\title{
Quantifiers in a Multimodal World: Hallucinating Vision with Language and Sound
}

\author{
Alberto Testoni \\ Sandro Pezzelle \\ CIMeC - University of Trento \\ ILLC - University of Amsterdam \\ alberto.testoni@studenti.unitn.it s.pezzelle@uva.nl
}

\author{
Raffaella Bernardi \\ CIMeC, DISI - University of Trento \\ raffaella.bernardi@unitn.it
}

\begin{abstract}
Inspired by the literature on multisensory integration, we develop a computational model to ground quantifiers in perception. The model learns to pick out of nine quantifiers ('few', 'many', 'all', etc.) the one that is more likely to describe the percent of animals in a visualauditory input containing both animals and artifacts. We show that relying on concurrent sensory inputs increases model performance on the quantification task. Moreover, we evaluate the model in a situation in which only the auditory modality is given, while the visual one is 'hallucinanted' either from the auditory input itself or from a linguistic caption describing the quantity of entities in the auditory input. This way, the model exploits prior associations between modalities. We show that the model profits from the prior knowledge and outperforms the auditory-only setting.
\end{abstract}

\section{Introduction}

Quantifiers (words like 'some', 'most', 'all') have long been the holy grail of formal semanticists (see Peters et al. (2006) for an overview). More recently, they have caught the attention of cognitive scientists, who showed that these expressions are handled by children quite early in life (Halberda et al., 2008), even before developing the ability to count (Hurewitz et al., 2006). Though some effort has been paid to model these highfrequency expressions from their use in big corpora of texts (Baroni et al., 2012; Herbelot and Vecchi, 2015), relatively little work has focused on the models' ability to quantify using these words.

In computer vision, some focus to the task of extracting quantities from images has been expressed through visual question answering, whose benchmark dataset (Antol et al., 2015) contains 'count questions' (e.g., 'How many Xs have the property Y?') that repeatedly turned out to be

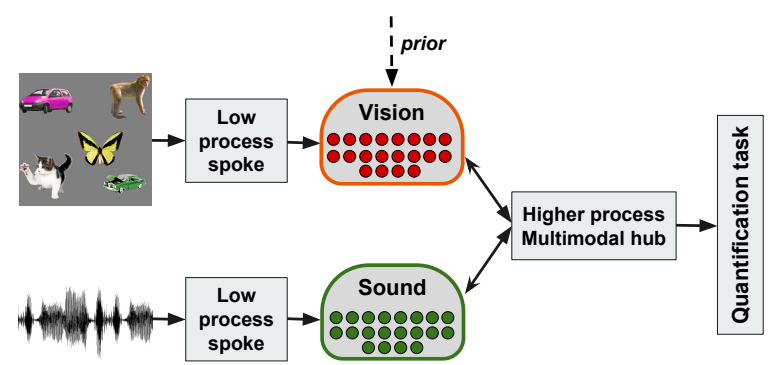

Figure 1: Learning to quantify through a 'Hub and Spoke' model enhanced with prior knowledge. The Hub learns to integrate multisensory inputs, whose representations (Spokes) are affected by such integration and can be 'hallucinated' by prior knowledge. We focus on how this prior knowledge hallucinates the visual representation (signalled by the dotted arrow).

rather challenging (Malinowski et al., 2015; Fukui et al., 2016). While this work paid little attention to quantifiers, a few recent studies specifically investigated their computational learning from visual inputs (Sorodoc et al., 2016; Pezzelle et al., 2017). These works built on the evidence that (part of) the meaning of quantifiers is grounded in perception. However, they only experimented with the visual modality, though the numerical representations humans derive from sensory inputs have been shown to be shared across modalities, e.g., vision and sound (Feigenson et al., 2004).

In the literature on multisensory integration it is well established that redundant information conveyed through different sensory inputs leads to a better performance on semantic tasks (McGurk and MacDonald, 1976). These findings have brought researchers to propose the 'Hub and Spoke' model (hence, H\&S): concepts are learned by mutual interaction of the representation produced by sensory specific processors, the 'spokes', with a transmodal 'hub' (Patterson et al., 2007; 
Ralph et al., 2017). The role of the cross-modal hub is to take each of the spokes' output and to reproduce the correct information across the others by back-propagation (Ralph et al., 2017). There is evidence that memory recall is affected by the multisensory context in which the concept was learned. In particular, it has been shown that a congruent pair of audiovisual inputs may facilitate subsequent recall. In other words, we learn to process a sound (e.g., 'meow' or 'woof') and to associate it to the visual representation of the entity we see making it, and this facilitates the recall of the corresponding concept (i.e., 'cat' or 'dog').

In this work, we apply the H\&S model to the conceptual learning of quantifiers and study how the hub learns to integrate the visual and auditory spoke representations (as illustrated in Figure 1) to perform the quantification task. That is, the model has to learn to say that 'none', 'few', 'most', etc. of the objects in the visual and auditory inputs belong to a given category, that of animals. We focus on 9 common quantifiers and experiment with visual and auditory inputs strongly aligned (viz., aligned at the entity level). We show that

- Using congruent audio visual inputs increases the performance of the model in learning quantifiers within single-sensory models;

- The H\&S model can generalize to unseen data quite well. In particular, it generalizes better when trained on small combinations and tested on large ones than vice versa.

Furthermore, a second part of our work is based on an ongoing debate in multisensory integration, namely whether the processing of sensory inputs is passive or rather influenced by previous experience that creates cross-sensory associations. Within this debate, one of the most influential frameworks is the Predictive Coding Model (hence, PCM), according to which prior knowledge affects the representation of perceptual inputs (Friston, 2010). There is a general agreement on the predictive effects between visual and auditory inputs, whereas the role of language in priming visual perception is still under debate (see Simanova et al. (2016) for an overview).

Inspired by this work, we compare a single auditory sensory model with a model in which the processing of the auditory stimuli is facilitated by prior expectation elicited by either the visual spoke (implemented as a mapping from the experienced auditory input to its corresponding visual representation) or the language input (again implemented as a mapping from language to visual representations). In Figure 1, the 'prior' arrow illustrates this predictive factor. Simplifying somewhat, we simulate a setting where a model, trained to quantify from co-occurring synchronous audio visual inputs, is tested on a situation where (a) it hears but does not see the entities (audio-vision association prior) or (b) it reads a description of the entities and hears their sounds but does not see them (language-vision association prior). We show that

- Using priors hallucinating the visual representation improves the performance of the model compared to when it receives only auditory inputs;

- Language prior is slightly more effective than sound prior to hallucinate concurring vision.

\section{Related Work}

\subsection{Multimodal Models}

Fueled by the explosion of deep learning, much effort has been paid in recent years to develop models that exploit information from various modalities. Attention has been mostly on language and vision, for which various tasks have been proposed, i.e. image captioning (Hodosh et al., 2013), visual question answering (Antol et al., 2015; Goyal et al., 2017), visual reasoning (Andreas et al., 2016; Johnson et al., 2017; Suhr et al., 2017), visual storytelling (Huang et al., 2016; Gonzalez-Rico and Fuentes-Pineda, 2018), and visual dialogue (De Vries et al., 2017). While all this work combines images with written text, some other studies employed spoken language to perform various tasks, such as image-audio retrieval (Chrupała et al., 2017; Harwath et al., 2018). Overall, these works repeatedly showed that combining information from language and vision leads to representations that are beneficial in virtually any task.

A relatively recent strand of research focused on the integration of visual and sound information, where the latter is, e.g., the 'roar' of a fast car (Owens et al., 2016, 2018; Zhao et al., 2018).

More akin to our work is Aytar et al. (2017), who jointly investigated language, vision, and sound. By training a deep convolutional network 
for aligned representation learning across the three modalities, they showed that the emerging alignment improved both retrieval and classification performance. Interestingly, their results also suggested that, even though the network was never exposed to pairs of sounds and text inputs during training, an alignment between these two modalities was learned, possibly due to the use of images as an internal 'bridge'. We explore the same three modalities studied by Aytar et al. (2017). However, we use different models and evaluation settings (to mimic the PCM) and tackle a different task, namely quantification.

\subsection{Computational Models of Quantification}

The task of quantification (in the broad sense of providing some quantitative information), has been largely explored in computer vision (Seguí et al., 2015; Zhang et al., 2015a; Arteta et al., 2016). In these works, the focus is to provide the exact number of objects in a scene, and only rarely it is inspired by cognitive abilities (Zhang et al., 2015b; Chattopadhyay et al., 2017). Similarly, in the visual question answering community, the so called 'number' questions are almost exclusively about cardinals, with some exceptions including generalized quantifiers like every or more than half (Suhr et al., 2017; Kuhnle et al., 2018).

Inspired by the cognitive skill of Approximate Number Sense (ANS) is instead Stoianov and Zorzi (2012), which tested hierarchical generative networks and showed that they learn ANS as a statistical property of images. Practically speaking, the model was able to compare one approximate 'numerosity' against another and to perform a more/less task. Similar high-level cognitive abilities are required to humans to use vague quantifiers such as few, many, or most, whose meaning is heavily dependent on contextual factors. Using visual scenes as context, a recent strand of work has focused on the computational learning of quantifiers with neural networks. One approach tackled the task in a visual question answering fashion (Sorodoc et al., 2018), while another aimed at learning to apply the correct quantifier to a given scene (Sorodoc et al., 2016; Pezzelle et al., 2017).

More related to our work is Pezzelle et al. (2018b), which tested a model in the task of predicting the probability of each quantifier to be used in a given scene. The network was trained with probabilities from human participants by Pezzelle et al. (2018a). We use the same human annotation but make two steps further: First, we also experiment with auditory inputs; second, we experiment with different settings inspired by the literature on multisensory integration.

\section{Task and Datasets}

\subsection{Task}

Given an input (a scene) consisting of entities that are either animals (targets) or artifacts (distractors), the model has to quantify the former. For instance, given the image in Figure 2 on the left, it should assign a high probability to 'most', whereas for the image on the right it should assign a high probability to 'few'. The inputs are either unimodal (sound, vision) or multimodal (sound+real vision, sound+hallucinated vision). We inherit and adapt to our multimodal datasets the gold standard annotation collected by Pezzelle et al. (2018a): Human participants were asked to select, out of nine quantifiers ('none', 'almost none', 'few', 'the smaller part', 'some', 'many', 'most', 'almost all', 'all'), the one that best referred to the set of animals depicted in a brieflypresented visual scene (these scenes were similar, but not identical to those in Figure 2). Each quantifier turned out to be used to refer to various proportions of animals. For instance, 'most' could apply when animals corresponded to $57 \%, 60 \%, 67 \%$, $75 \%$ and $80 \%$ of the objects. At the same time, various proportions had different probabilities to be referred by a given quantifier. With a proportion of $60 \%$ animals, for example, the probability to choose 'most', 'many' and 'some' is $0.52,0.20$ and 0.18 , respectively. The models have to learn the probability distribution associated with each proportion. Intuitively, 'none' and 'all' are almost exclusively used with, respectively, $0 \%$ and $100 \%$ animals.

\subsection{Datasets}

Following Pezzelle et al. (2018a), our datasets consist of scenes containing animals and artifacts with a minimum of 3 and a maximum of 20 entities in total. There are in total 17 proportions, out of which 8 contain more animals than artifacts, 8 contain more artifacts than animals, and 1 contains an equal number of them. ${ }^{1}$ For each proportion

\footnotetext{
${ }^{1}$ The proportions obtained by having min. 3 max $20 \mathrm{ob}-$ jects are: $0 \%, 10 \%, 17 \%, 20 \%, 25 \%, 33 \%, 40 \%, 43 \%, 50 \%$, $57 \%, 60 \%, 67 \%, 75 \%, 80 \%, 83 \%, 90 \%, 100 \%$.
} 

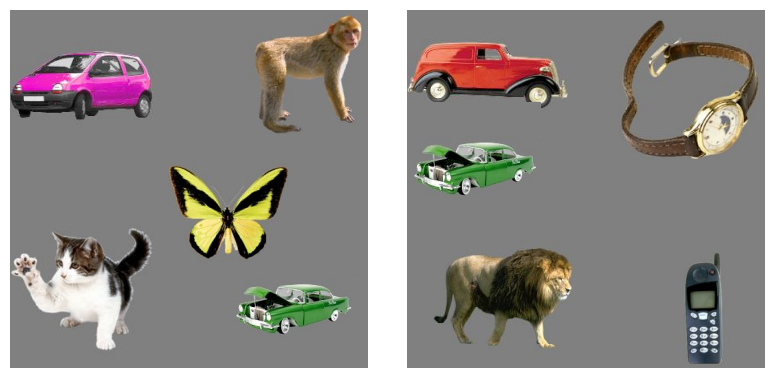

Figure 2: Visual dataset. Left: 'most' $(60 \%)$ of the objects are animals, viz. 3:2. Right: 'few' (20\%) of the objects are animals, viz. 1:4.

we generated scenes containing all possible combinations of cardinalities: For the proportion 0\%, for example, 17 combinations were built, ranging from 0:3 (0 animals, 3 artifacts) to 0:20.

We built visual and auditory datasets aligned at the entity level: For each image, we created the corresponding auditory datapoint containing the sound of each entity in the image. By so doing, using the terminology of (Aytar et al., 2018), we obtained strongly aligned visual and auditory datasets. In total, we used 55 unique animals and 55 unique artifacts. We only used those entities for which we could have whole-depicting images (not just parts) and for which we had a corresponding sound. Furthermore, for each audio-visual input we created a corresponding linguistic caption describing the quantities of the entities in it. Details on the three datasets are provided below.

Visual Dataset Similarly to Pezzelle et al. (2018b), we built a large dataset of synthetic visual scenes depicting a variable number of animals and artifacts on top of a neutral grey background (see Figure 2). The scenes were automatically generated using the following pipeline: (a) Natural images depicting target objects (e.g., a dog) or distractors (e.g., a car) were randomly picked up from the 110 entities pre-selected from the dataset by Kiani et al. (2007). As opposed to the synthetic dataset of Pezzelle et al. (2018a), where multiple copies of the same animal/artifact were reproduced in the scene, we have different target/distractor instances in each scenario (e.g, different instances of 'car' as in Figure 2 (right)). However, we do not vary the size and orientation of entities; (b) The proportion of targets in the scene was chosen by selecting only those matching the 17 pre-defined proportions mentioned above. We generated $17 \mathrm{~K}$ scenes balanced

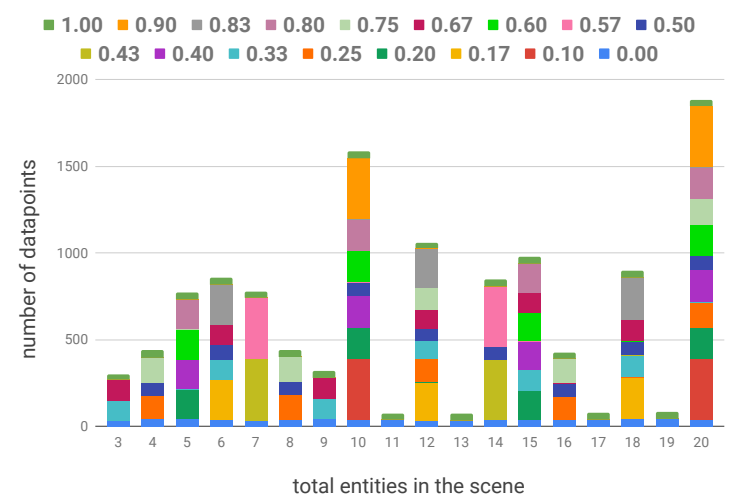

Figure 3: Histogram representing the number of total objects in the scene for the 17 different proportions (training set). On top the 17 proportions.

per proportion (1K scenes/proportion), and split them into train $(70 \%)$, validation $(10 \%)$, and test (20\%) sets. The distribution of proportions per total number of objects in the training set is illustrated in Figure 3.

Auditory Dataset We followed a similar procedure to build the auditory scenes. We took Audioset (Gemmeke et al., 2017) as our starting point to obtain sounds corresponding to the entities since it contains a huge collection of humanlabeled 10-sec sound clips. It is organized as a hierarchical graph of event categories, covering a wide range of human and animal sounds, musical instruments and genres, and common everyday environmental sounds. We took sounds belonging to the categories of 'animals' and 'tools'. We built our auditory dataset starting from the visual one described above and obtained the strongly aligned auditory version. Hence, as in the case of the visual datapoint, an auditory datapoint can contain different instances of the same type of animal/artifact. The auditory dataset consists of $17 \mathrm{~K}$ scenes again balanced per proportion $(1 \mathrm{~K}$ scenes/proportion), with the same split as the visual one and each 'scene' containing min 3 max 20 entities out of 110 entities.

Linguistic Dataset For each aligned visual and auditory input pair, we built a linguistic caption describing the exact quantities of the entities present in it (for instance, for the image in Figure 2 (left), we obtain 'There are one butterfly, two automobiles and two mammals'). The procedure, illustrated in Figure 4, is as following: (a) We manually annotated each of the 110 entities used to 


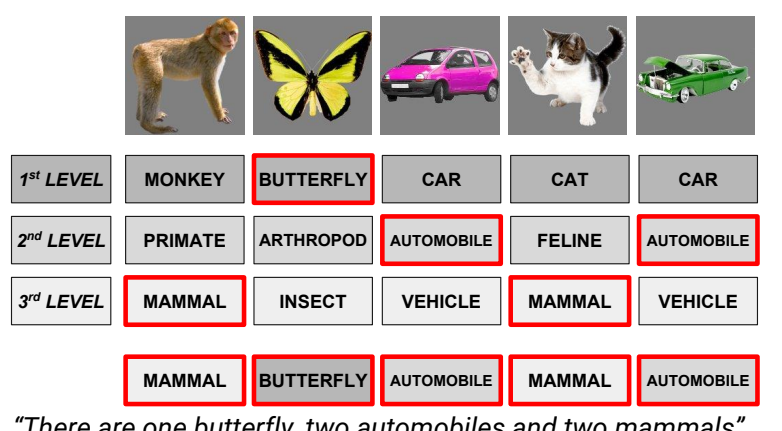

"There are one butterfly, two automobiles and two mammals"

Figure 4: Linguistic dataset construction. In red: randomly selected nouns for each entity. Bottom: generated caption.

build the dataset (55 animals and 55 artifacts) with 3 nouns expressing different levels of an ontological hierarchy (e.g., 'cat', 'feline', 'mammal'). ${ }^{2}$ (b) For each entity present in the audio-visual scene, we randomly picked one of the three nouns. (c) For each noun, we counted the number of entities present in the audio-visual input, assigned that number to the noun and pluralized it, if necessary. (d) In order to account for more variability, we started the linguistic caption by choosing one of six possible starting phrases. ${ }^{3}$ We obtained captions with on average 10.5 nouns (standard deviation: 4.53).

Sensory Representations The vector representation of the visual scene is extracted using Inception v3 CNN (Szegedy et al., 2016) pretrained on ImageNet (Deng et al., 2009) from the last average pooling layer which consists of 2048-d visual vectors.

For the auditory dataset, we built the representation of each entity and the scenes containing them as following. We started from the audio features computed with the VGG-inspired auditory model described in Hershey et al. (2017) which has been trained on a preliminary version of YouTube-8M. ${ }^{4}$ For each second of a sound clip, the model produces a 128-d vector; hence each 10sec sound clip of the Audioset dataset (Gemmeke et al., 2017) would be represented by a 1280-d

\footnotetext{
${ }^{2}$ Note that in the case of animals, this hierarchy is much more easier to build (e.g. Linnaean taxonomy) while for the artifacts the 3 nouns are generally more often synonyms and often do not represent a real hierarchy/taxonomy.

3 'There are ...', , 'It seems to me that there are ... ', 'I'm thinking of ...', 'I can spot ...', 'There exists ...', 'I can spot ...').

${ }^{4}$ https: / / research.google.com/ youtube $8 \mathrm{~m} /$
}

vector. To work with smaller and more representative vectors, we selected the two central seconds of each 10-sec audio clip (the 5th and 6th) and used the resulting 256-d vector as the representation of the corresponding entity. Out of these entity representations we built the representation of the scene by concatenating the entity vectors. Scenes can contain min 3 and max 20 entities, hence we use vectors of 20 'cells'. When there are less than 20 entities, there are 'empty cells' which are visually represented by the grey background. We represented their auditory counterpart with a 'silent sound' computed as following: we recorded a 10sec sound clip of silence, picked the 5th and 6th seconds and obtained the 256-d auditory vector using the model of Hershey et al. (2017). The 20 total 'cells' are then shuffled, resulting in a 5120-d auditory vector.

As for the linguistic scenes, for each caption we extracted the features through the Universal Sentence Encoder (USE) (Cer et al., 2018) producing 512 dimensional vectors for each sentence. Alternatively, we could have used LSTM modules to process from scratch both the linguistic and acoustic inputs exploiting their sequential nature. We rejected this alternative mainly to avoid that, during the training process, the neural network learns task-dependent representations and arbitrary associations. It has been shown (e.g., in Cer et al. (2018)) that USE provides sentence-level embeddings with strong transfer performance on several NLP tasks. We consider this point as a strong motivation for our choice: in this way, we get more consistent representations across different modalities and the overall architecture turns out to be easier, more scalable and less prone to learn taskspecific representations.

The semantic spaces containing the entity representations of the three modalities are rather different. It is interesting to note that the auditory dataset is much more dense than either the visual or the linguistic one: The average cosine similarity between entity pairs is 0.73 for sound vs. 0.44 for vision and 0.43 for language. In other words, entities are visually and linguistically much more distinct than auditorily. This could be possibly due to the fact that, as highlighted by Owens et al. (2018), sound undergoes less transformations than vision, which is affected by, for instance, lighting, scene composition, and viewing angle. In other words, sound could be denser than vision since it 
'abstracts' from all the possible visual transformations that we encounter in the other modality. It follows that integrating these modalities requires some degree of generalization over a variety of transformations, which is intuitively not trivial.

\section{Models and Test Settings}

Below we describe the 'Hub and Spoke' model $(H \& S)$ that takes as input strongly aligned auditory and visual inputs, and the 'Predictive Code Model' (PCM) which differs from the former only at testing time, when it takes as input the vector processed by the auditory spoke and the visual representation obtained by prior knowledge, viz. through an external mapping. We take as baselines the single-modality (visual, auditory inputs) versions of the model.

Hub and Spoke model (H\&S) As illustrated in Figure 5 (up), this model takes the 2048-d and 5120 -d visual and auditory vectors, reduces them to vectors of the same dimensions (512-d) and merges them in the Hub through multiplication. The multimodal output is reduced to 128 -d via a ReLU hidden layer, then a softmax layer is applied to output a 9-d vector with the probabilities to assign each of the 9 quantifiers.

Unimodal model The three layers of the hub described above are trained to perform the quantification task from either the visual or auditory representations alone.

Predictive Code Model (PCM) We take the hub trained using the representations produced by the visual and auditory spokes (namely the hub of the $H \& S$ ) and evaluate it on new types of audio-visual inputs: the auditory vectors are produced by the auditory spoke as for the $\mathrm{H} \& \mathrm{~S}$, while the visual vectors are obtained via a linear mapping function that simulates prior knowledge which 'hallucinates' the visual perception. The mapping function takes as input either (a) the auditory input itself (auditory prior) or (b) the corresponding linguistic caption (language prior), as illustrated in Figure 5 (bottom, (a) left vs. (b) right). For sake of simplicity, the mapping function is trained outside the model. It is implemented as a linear neural network which is exposed to the aligned data of the training and validation sets used for the H\&S. Hence, when used in the PCM setting it is applied to data that was never seen before. The mapping is trained using Mean Squared Error (MSE).
We only experimented with hallucinated visual representations and left for the future the other direction - a visual experience facilitated by the corresponding imagined auditory. Since the semantic space of the auditory input is rather dense, we expect that a non-linear mapping might be necessary to obtain the latter.

Implementation details We used ReLU activation function for all the hidden layers, and Adam optimizer (Kingma and $\mathrm{Ba}, 2015$ ) with learning rate $=0.0001$ and default weight decay. All models were trained for no more than 150 epochs (using early stopping) by minimizing the KullbackLeibler (KL) divergence loss between the activations by softmax and the probability distribution of human responses for each proportion by Pezzelle et al. (2018a). All models were implemented in PyTorch v0.4.

\section{Experiments and Results}

Evaluation All models are evaluated by computing the Pearson product-moment correlation coefficient between the Softmax probabilities and the 9-d vectors from Pezzelle et al. (2018a), which encode the probability of each quantifier to be used with respect to a given proportion based on human choices.

\subsection{Experiments}

Unimodal vs. multimodal models Testing the models on the unimodal and multimodal data might lead to results that are influenced by the different sizes of data seen during training. To rule out this possibility, we use unimodal and multimodal datasets of equal size. We take 11,900 datapoints for each single modality; and in the multimodal model, we use 5950 instances for each modality which sum up to 11,900 datapoints.

Incongruent visual-auditory inputs In order to test the effectiveness of the integration of the two modalities, we take the H\&S trained on aligned (congruent) visual-auditory data and we test it with incongruent data, viz. inputs that do not have the same proportion of animals. Given a visual input containing, e.g., 3 animals and 2 artifacts (as in Figure 2 left), we pair it with an auditory input having 3 artifacts and 2 animals. This way, the corresponding probability distributions are different, hence we refer to these pairs as incongruent auditory input. Similarly, we generate incongruent vi- 

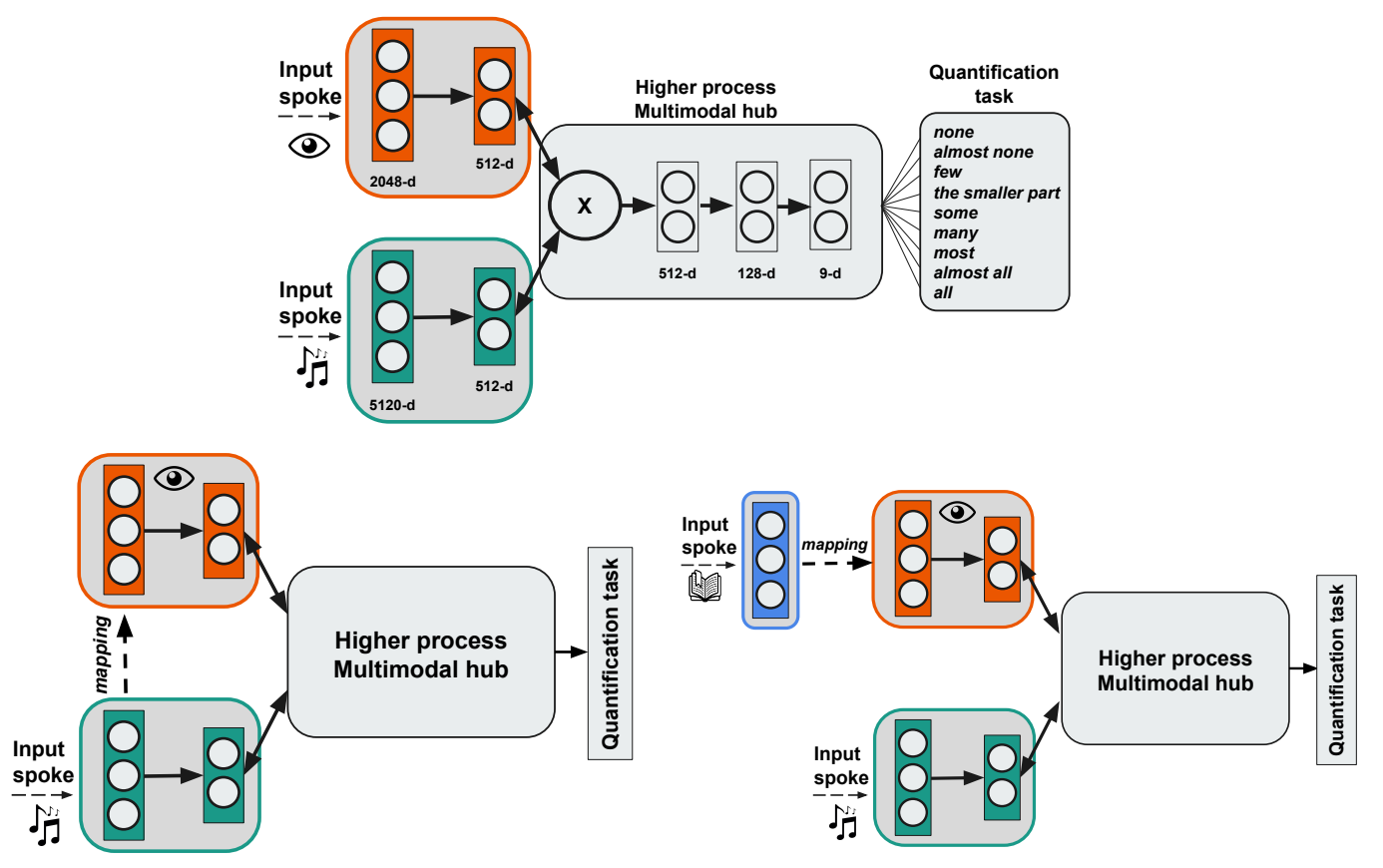

Figure 5: Up: H\&S To learn quantifiers, the hub learns to integrate the auditory and visual sensory inputs; Bottom: PCM The hub trained to perform audio-visual integration can quantify the animals present in the auditory inputs by exploiting the 'hallucinated' visual representation obtained either from (a) the auditory input it self (left corner) or (b) the the language input (right corner).

sual inputs by pairing an auditory input with, e.g, a 3:2 proportion with a visual input with a proportion of $2: 3$, and consider as the correct probability distribution the one corresponding to the $3: 2$ proportion encoded by the auditory input. To ensure that the difference between the two modalities is high, we avoid pairing proportions with extremely similar probability distributions. Rather, we focus on a subset of proportion pairs, namely $0-100 \%$, $10-90 \%$, and $17-83 \%$. If the hub exploits the alignment between the modalities, we expect the model to perform poorly in this setting (lower is better).

Unseen combinations We evaluate the generalization power of the models by testing them on unseen data. We want to study how well the model generalizes from (a) small cardinalities to larger ones and (b) vice versa. To this end, we divide the training and test sets as following: For each of the 17 proportions, we use as the test set the scenes containing (a) the largest possible number of objects (e.g., for proportion $0 \%$, we test on 0:20 and train on all the other combinations); (b) the smallest possible number of objects (e.g., for proportion $0 \%$, we test on $0: 3$ and train on all the other combinations).

\begin{tabular}{l|c} 
& Pearson's $r$ \\
\hline Sound & 0.68 \\
Vision & 0.72 \\
\hline H\&S & 0.86 \\
PCM: auditory prior & 0.78 \\
PCM: language prior & 0.81 \\
\hline H\&S on incongruent visual inputs & -0.25 \\
H\&S on incongruent auditory inputs & 0.02
\end{tabular}

Table 1: Pearson's r correlation results - human judgments used as target results. Unimodal vs. multimodal model trained and tested on datasets of equal size.

\subsection{Quantitative Results}

Unimodal vs. multimodal models Table 1 reports the Pearson's $r$ correlation results comparing the unimodal and multimodal models. As we can see, the visual data is slightly more informative than the auditory one for learning the quantification task ( 0.68 vs. 0.72$)$. The first main result is that the multimodal model outperforms the unimodal ones to a large extent. The H\&S obtains 0.18 and 0.14 higher correlation than the auditory and visual model, respectively. This result shows that the multimodal data provide complementary information that the model manages to exploit. Regarding the effect of prior knowledge, 


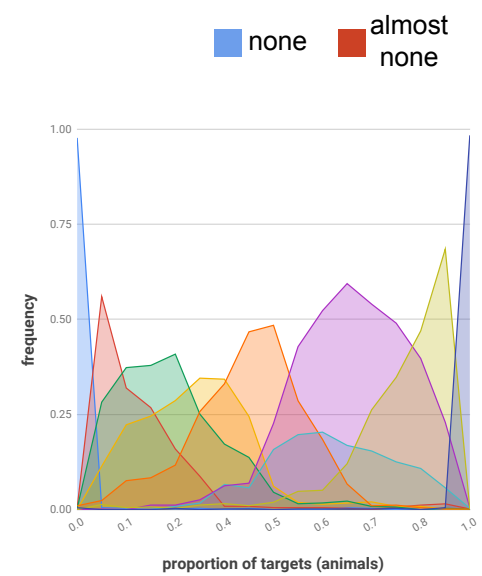

A. Humans

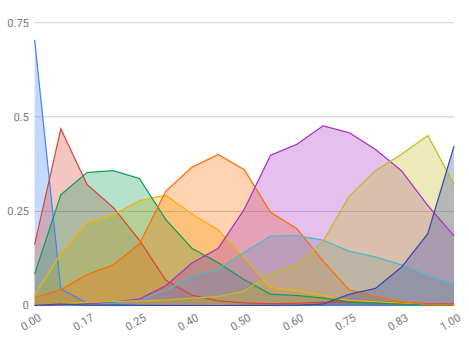

proportion of targets (animals)

D. $H \& S$

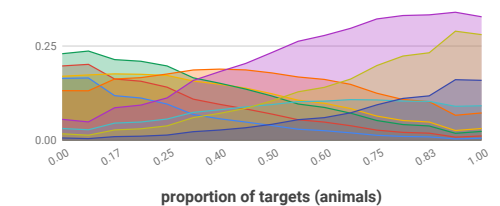

B. Sound unimodal

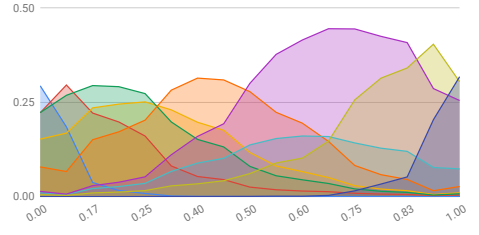

proportion of targets (animals)

E. PCM Sound prior

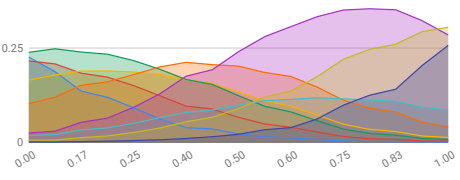

proportion of targets (animals)

C. Vision unimodal

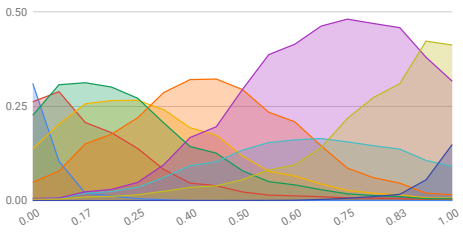

proportion of targets (animals)

F. PCM Language prior

Figure 6: A: Density plot reporting the frequency of human responses for the 9 quantifiers (y-axis) against the proportion of targets in the scene (x-axis). B-F: Average probabilities predicted by models in test set (same axes).

\begin{tabular}{l|cc} 
& \multicolumn{2}{|c}{ Pearson's $r$} \\
& large $\rightarrow$ small & small $\rightarrow$ large \\
\hline Sound & 0.55 & 0.73 \\
Vision & 0.64 & 0.76 \\
\hline H\&S & 0.74 & 0.85
\end{tabular}

Table 2: Unimodal vs. multimodal models tested on unseen combinations which have smaller or larger number of entities than the seen data.

we see that hallucinating the visual representations improves over processing only the auditory input. Using the latter to hallucinate the visual scene leads to an increase of 0.10 in correlation, and an even higher increase $(+0.13)$ is obtained when the hallucination is induced by a linguistic description of the scene. It is worth noticing, however, that the correlation values obtained by the PCMs are slightly lower than the one obtained by the H\&S. This is intuitive since the latter can capitalize on first-hand information from both modalities.
To better understand the behavior of the multimodal model, we scrutinize its results by investigating whether the absolute difference between the animals and artifacts sets has an impact on the performance of the model. Figure 7 reports Pearson's $r$ obtained by the H\&S model for the smallest and highest combination of each proportion (we do not plot proportion 0.5 since the distance is 0 for all its combinations). For instance, for proportion $67 \%$, the smallest combination is $2 / 3$ ( 2 targets, 1 nontargets), the largest combination is $12 / 18(12,6)$, and their absolute difference is equal to 1 and 6 , respectively. As can be seen from the plot, smaller absolute differences are always harder than higher ones.

Incongruent sensory pairs As the results in Table 1 show, the model is strongly sensitive to these incongruent data, suggesting that cross-modal integration is actually part of the models. 


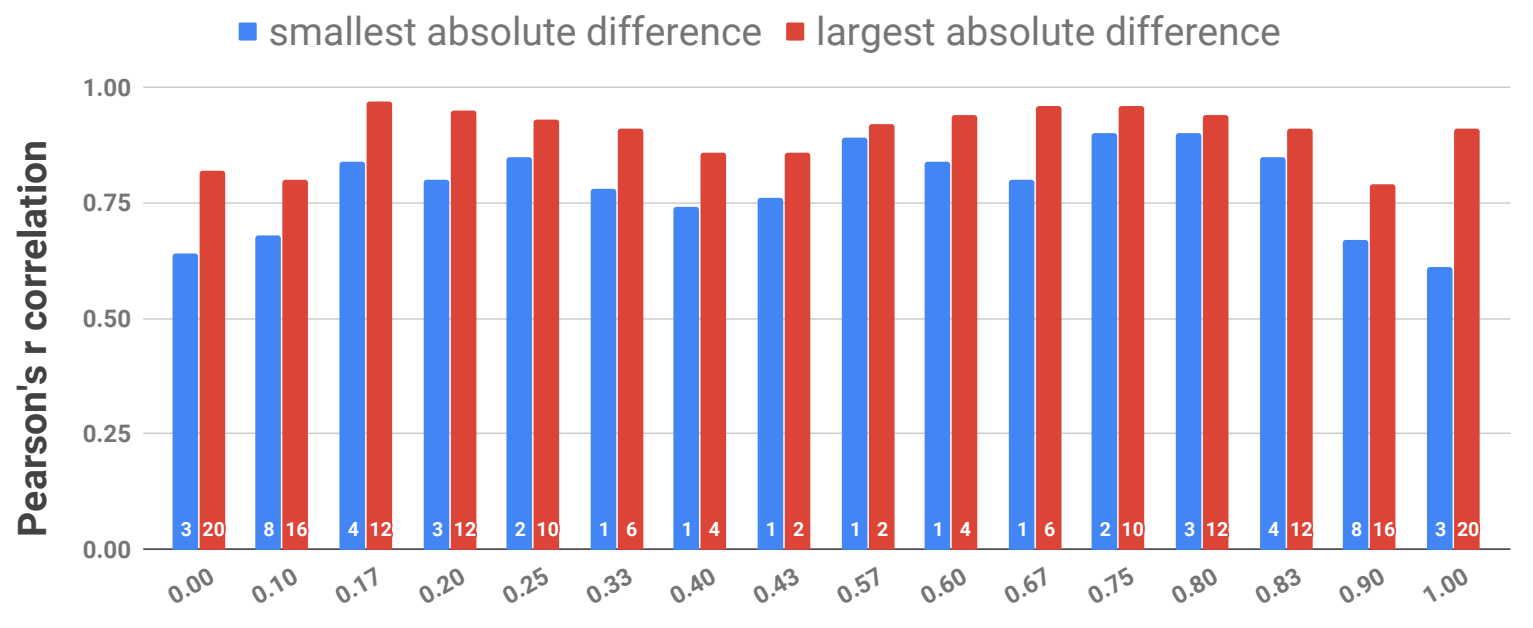

Proportion of animals in the scene

Figure 7: H\&S Pearson's $r$ obtained for the smallest (blue) and biggest (red) combination of each proportion. Note that numbers in white at the bottom of each bar refer to the absolute difference between animals and artifacts sets.

Unseen combinations Table 2 shows that models are able to generalize to unseen combinations quite well. In particular, they turn out to be always better in generalization when they learn from small combinations and are tested on large ones. This pattern of results reflects the findings illustrated in Figure 7, assuming that a model trained on hard cases and tested on easier ones would lead to higher results compared to the opposite 'direction'.

\subsection{Qualitative Results}

Figure 6 compares the probability distributions learned by the tested models (panels B-F) against the distribution of responses by humans (panel A) from Pezzelle et al. (2018a). As can be clearly seen, both unimodal models (B-C) show a much lower correlation with human data compared to either H\&S (D) or PCMs (E-F). In particular, the unimodal models tend to produce very similar curves for all quantifiers, thus predicting them with a similar probability at any proportion (i.e., there are no clear 'peaks'). Both the H\&S and the PCMs, in contrast, output a distribution that is very similar to that by humans (mirrored in the results of Table 1). While plots D-F are almost identical, it can be noted that the H\&S is slightly better than both PCMs at the 'extreme' proportions, particularly $0 \%$ and $100 \%$. We conjecture this ability is responsible of the slightly higher correlation obtained by this model compared to the PCMs.

\section{Conclusion}

In this paper, we show that concurrent multisensory information bootstraps models performance in a semantic task, namely grounding quantifiers, in line with the results on human perception. Also, we provide computational evidence that the predicting code hypothesis advocated in the cognitive literature is an interesting and useful source of inspiration for computational models. We plan to further investigate how predictions from prior knowledge can be compared with those obtained through sensory experience to further improve the performance on semantic tasks.

\section{Acknowledgements}

We kindly acknowledge the support of NVIDIA Corporation with the donation to the University of Trento of the GPUs used in our research. We thank Aurélie Herbelot, Manuela Piazza, and Marco Marelli for their valuable comments. The second author is funded by the Netherlands Organisation for Scientific Research (NWO) under VIDI grant no. 276-89-008, Asymmetry in Conversation.

\section{References}

Jacob Andreas, Marcus Rohrbach, Trevor Darrell, and Dan Klein. 2016. Neural module networks. In Proceedings of the IEEE Conference on Computer Vision and Pattern Recognition, pages 39-48. 
Stanislaw Antol, Aishwarya Agrawal, Jiasen Lu, Margaret Mitchell, Dhruv Batra, C Lawrence Zitnick, and Devi Parikh. 2015. Vqa: Visual question answering. In Proceedings of the IEEE international conference on computer vision, pages 2425-2433.

Carlos Arteta, Victor Lempitsky, and Andrew Zisserman. 2016. Counting in the wild. In European Conference on Computer Vision, pages 483-498. Springer.

Yusuf Aytar, Lluis Castrejon, Carl Vondrick, Hamed Pirsiavash, and Antonio Torralba. 2018. Crossmodal scene networks. IEEE transactions on pattern analysis and machine intelligence, 40(10):2303-2314.

Yusuf Aytar, Carl Vondrick, and Antonio Torralba. 2017. See, hear, and read: Deep aligned representations. arXiv preprint arXiv:1706.00932.

Marco Baroni, Raffaella Bernardi, Ngoc-Quynh Do, and Chung-chieh Shan. 2012. Entailment above the word level in distributional semantics. In Proceedings of the 13th Conference of the European Chapter of the Association for Computational Linguistics, pages 23-32. Association for Computational Linguistics.

Daniel Cer, Yinfei Yang, Sheng-yi Kong, Nan Hua, Nicole Limtiaco, Rhomni St John, Noah Constant, Mario Guajardo-Cespedes, Steve Yuan, Chris Tar, et al. 2018. Universal sentence encoder. arXiv preprint arXiv:1803.11175.

Prithvijit Chattopadhyay, Ramakrishna Vedantam, Ramprasaath R. Selvaraju, Dhruv Batra, and Devi Parikh. 2017. Counting everyday objects in everyday scenes. In The IEEE Conference on Computer Vision and Pattern Recognition (CVPR).

Grzegorz Chrupała, Lieke Gelderloos, and Afra Alishahi. 2017. Representations of language in a model of visually grounded speech signal. In Proceedings of the 55th Annual Meeting of the Association for Computational Linguistics (Volume 1: Long Papers), volume 1, pages 613-622.

Harm De Vries, Florian Strub, Sarath Chandar, Olivier Pietquin, Hugo Larochelle, and Aaron C Courville. 2017. GuessWhat?! Visual object discovery through multi-modal dialogue. In $C V P R$, volume 1 , page 3 .

Jia Deng, Wei Dong, Richard Socher, Li-Jia Li, Kai Li, and Li Fei-Fei. 2009. Imagenet: A large-scale hierarchical image database. In Computer Vision and Pattern Recognition, 2009. CVPR 2009. IEEE Conference on, pages 248-255. Ieee.

Lisa Feigenson, Stanislas Dehaene, and Elizabeth Spelke. 2004. Core systems of number. Trends in cognitive sciences, 8(7):307-314.
K. Friston. 2010. The free-energy principle: an unified brain theory? Thefreeenergyprinciple:aunifiedbraintheory?, 11:127-138. Doi:10.1038/nrn2787.

Akira Fukui, Dong Huk Park, Daylen Yang, Anna Rohrbach, Trevor Darrell, and Marcus Rohrbach. 2016. Multimodal compact bilinear pooling for visual question answering and visual grounding. In Proceedings of the 2016 Conference on Empirical Methods in Natural Language Processing, pages 457-468.

Jort F Gemmeke, Daniel PW Ellis, Dylan Freedman, Aren Jansen, Wade Lawrence, R Channing Moore, Manoj Plakal, and Marvin Ritter. 2017. Audio set: An ontology and human-labeled dataset for audio events. In Acoustics, Speech and Signal Processing (ICASSP), 2017 IEEE International Conference on, pages 776-780. IEEE.

Diana Gonzalez-Rico and Gibran Fuentes-Pineda. 2018. Contextualize, Show and Tell: A Neural Visual Storyteller. arXiv preprint arXiv:1806.00738.

Yash Goyal, Tejas Khot, Douglas Summers-Stay, Dhruv Batra, and Devi Parikh. 2017. Making the V in VQA matter: Elevating the role of image understanding in Visual Question Answering. In CVPR, volume 1 , page 3 .

Justin Halberda, Len Taing, and Jeffrey Lidz. 2008. The development of "most" comprehension and its potential dependence on counting ability in preschoolers. Language Learning and Development, 4(2):99-121.

David Harwath, Adrià Recasens, Dídac Surís, Galen Chuang, Antonio Torralba, and James Glass. 2018. Jointly discovering visual objects and spoken words from raw sensory input. In Proceedings of the European Conference on Computer Vision (ECCV), pages 649-665.

Aurélie Herbelot and Eva Maria Vecchi. 2015. Building a shared world: Mapping distributional to model-theoretic semantic spaces. In Proceedings of the 2015 Conference on Empirical Methods in Natural Language Processing, pages 22-32.

Shawn Hershey, Sourish Chaudhuri, Daniel PW Ellis, Jort F Gemmeke, Aren Jansen, R Channing Moore, Manoj Plakal, Devin Platt, Rif A Saurous, Bryan Seybold, et al. 2017. Cnn architectures for largescale audio classification. In Acoustics, Speech and Signal Processing (ICASSP), 2017 IEEE International Conference on, pages 131-135. IEEE.

Micah Hodosh, Peter Young, and Julia Hockenmaier. 2013. Framing image description as a ranking task: Data, models and evaluation metrics. Journal of Artificial Intelligence Research, 47:853-899.

Ting-Hao Kenneth Huang, Francis Ferraro, Nasrin Mostafazadeh, Ishan Misra, Aishwarya Agrawal, Jacob Devlin, Ross Girshick, Xiaodong He, Pushmeet 
Kohli, Dhruv Batra, et al. 2016. Visual storytelling. In Proceedings of the 2016 Conference of the North American Chapter of the Association for Computational Linguistics: Human Language Technologies, pages 1233-1239.

Felicia Hurewitz, Anna Papafragou, Lila Gleitman, and Rochel Gelman. 2006. Asymmetries in the acquisition of numbers and quantifiers. Language learning and development, 2(2):77-96.

Justin Johnson, Bharath Hariharan, Laurens van der Maaten, Li Fei-Fei, C Lawrence Zitnick, and Ross Girshick. 2017. CLEVR: A diagnostic dataset for compositional language and elementary visual reasoning. In Computer Vision and Pattern Recognition (CVPR), 2017 IEEE Conference on, pages 19881997. IEEE.

Roozbeh Kiani, Hossein Esteky, Koorosh Mirpour, and Keiji Tanaka. 2007. Object category structure in response patterns of neuronal population in monkey inferior temporal cortex. Journal of neurophysiology, 97(6):4296-4309.

Diederick P Kingma and Jimmy Ba. 2015. Adam: A method for stochastic optimization. In International Conference on Learning Representations (ICLR).

Alexander Kuhnle, Huiyuan Xie, and Ann Copestake. 2018. How clever is the FiLM model, and how clever can it be? In European Conference on Computer Vision, pages 162-172. Springer.

Mateusz Malinowski, Marcus Rohrbach, and Mario Fritz. 2015. Ask your neurons: A neural-based approach to answering questions about images. In Proceedings of the IEEE international conference on computer vision, pages 1-9.

H. McGurk and J. MacDonald. 1976. Hearing lips and seeing voices. Nature, 264:746-748. Doi:10.1038/264746a0.

Andrew Owens, Jiajun Wu, Josh H McDermott, William T Freeman, and Antonio Torralba. 2016. Ambient sound provides supervision for visual learning. In European Conference on Computer Vision, pages 801-816. Springer.

Andrew Owens, Jiajun Wu, Josh $\mathrm{H}$ McDermott, William T Freeman, and Antonio Torralba. 2018. Learning sight from sound: Ambient sound provides supervision for visual learning. International Journal of Computer Vision, 126(10):1120-1137.

Karalyn Patterson, Peter J Nestor, and Timothy T Rogers. 2007. Where do you know what you know? the representation of semantic knowledge in the human brain. Nature Reviews Neuroscience, 8(12):976.

Stanley Peters, , and Dag Westerståhl. 2006. Quantifiers in language and logic. Oxford University Press.
Sandro Pezzelle, Raffaella Bernardi, and Manuela Piazza. 2018a. Probing the mental representation of quantifiers. Cognition, 181:117-126.

Sandro Pezzelle, Marco Marelli, and Raffaella Bernardi. 2017. Be precise or fuzzy: Learning the meaning of cardinals and quantifiers from vision. In Proceedings of the 15th Conference of the European Chapter of the Association for Computational Linguistics: Volume 2, Short Papers, pages 337-342, Valencia, Spain. Association for Computational Linguistics.

Sandro Pezzelle, Ionut-Teodor Sorodoc, and Raffaella Bernardi. 2018b. Comparatives, quantifiers, proportions: a multi-task model for the learning of quantities from vision. In Proceedings of the 2018 Conference of the North American Chapter of the Association for Computational Linguistics: Human Language Technologies, Volume 1 (Long Papers), pages 419-430. Association for Computational Linguistics.

Matthew A Lambon Ralph, Elizabeth Jefferies, Karalyn Patterson, and Timothy T Rogers. 2017. The neural and computational bases of semantic cognition. Nature Reviews Neuroscience, 18(1):42.

Santi Seguí, Oriol Pujol, and Jordi Vitria. 2015. Learning to count with deep object features. In Proceedings of the IEEE Conference on Computer Vision and Pattern Recognition Workshops, pages 90-96.

Irina Simanova, Jolien C Francken, Floris P de Lange, and Harold Bekkering. 2016. Linguistic priors shape categorical perception. Language, Cognition and Neuroscience, 31(1):159-165.

Ionut Sorodoc, Angeliki Lazaridou, Gemma Boleda, Aurélie Herbelot, Sandro Pezzelle, and Raffaella Bernardi. 2016. "Look, some green circles!": Learning to quantify from images. In Proceedings of the 5th Workshop on Vision and Language, pages 75-79.

Ionut Sorodoc, Sandro Pezzelle, Aurélie Herbelot, Mariella Dimiccoli, and Raffaella Bernardi. 2018. Learning quantification from images: A structured neural architecture. Natural Language Engineering, page 1-30.

Ivilin Stoianov and Marco Zorzi. 2012. Emergence of a 'visual number sense' in hierarchical generative models. Nature neuroscience, 15(2):194-196.

Alane Suhr, Mike Lewis, James Yeh, and Yoav Artzi. 2017. A corpus of natural language for visual reasoning. In Proceedings of the 55th Annual Meeting of the Association for Computational Linguistics (Volume 2: Short Papers), volume 2, pages 217-223.

Christian Szegedy, Vincent Vanhoucke, Sergey Ioffe, Jon Shlens, and Zbigniew Wojna. 2016. Rethinking the inception architecture for computer vision. In Proceedings of the IEEE conference on computer vision and pattern recognition, pages 2818-2826. 
Cong Zhang, Hongsheng Li, Xiaogang Wang, and Xiaokang Yang. 2015a. Cross-scene crowd counting via deep convolutional neural networks. In Proceedings of the IEEE Conference on Computer Vision and Pattern Recognition, pages 833-841.

Jianming Zhang, Shugao Ma, Mehrnoosh Sameki, Stan Sclaroff, Margrit Betke, Zhe Lin, Xiaohui Shen, Brian Price, and Radomir Mech. 2015b. Salient object subitizing. In Proceedings of the IEEE Conference on Computer Vision and Pattern Recognition, pages 4045-4054.

Hang Zhao, Chuang Gan, Andrew Rouditchenko, Carl Vondrick, Josh McDermott, and Antonio Torralba. 2018. The sound of pixels. In Proceedings of the European Conference on Computer Vision (ECCV), pages 570-586. 